\title{
ON THE BINARY NATURE OF THE SLOW NOVA, RR TELESCOPII
}

\author{
B. L. WEBSTER
}

Royal Greenwich Observatory, Hailsham, Sussex, England

\begin{abstract}
Those novae known to be binaries generally have orbital periods of the order of hours, exceptions being the atypical recurrent novae T CrB and RS Oph, which have giant companions and probably much longer periods. Since the orbital period in a semi-detached system relates to the mechanism of current mass exchange and also to the extent to which the primary evolved before mass exchange took place at an earlier stage, it is of interest to see if any classical novae are in more widely separated systems.

This communication concerns the star RR Telescopii, which has all the characteristics of a slow nova - a range in amplitude greater than seven magnitudes, a spectral type at maximum of $F$, and a decline through a nebular stage of increasing ionization level (e.g. Thackeray, 1955). RR Tel was seen as a variable before outburst, but little is known about this variable apart from its period of 387 days, although doubts have been expressed about its being a red variable (Payne-Gaposchkin, 1957). Dr Thackeray has Radcliffe spectrograms of RR Tel from soon after maximum to the present. On some of the more recent of these, bands of TiO have become visible, presumably as the hot star has faded, and Dr Thackeray and I interpret these as meaning that the original variable is still there and is an M giant. Thus RR Tel is in a binary system containing a giant $\mathbf{M}$ star and a hot companion and such a system cannot have an orbital period of hours like the classical novae mentioned above.

In 1972, Dr Glass and I examined RR Tel in the infrared between 1.2 and $20 \mu$. The energy distribution does not resemble the cool star that might be expected, but is exactly like that of free-free radiation over the whole wavelength range. The puzzle is that the infrared is two orders of magnitude stronger than we would predict from the optical spectrum for free-free radiation.
\end{abstract}

\section{References}

Payne-Gaposchkin, C.: 1957, in J. H. Oort, M. G. J. Minnaert, and H. C. van de Hulst (eds.), The Galactic Novae, North-Holland Publishing Company, Amsterdam, p. 157.

Thackeray, A. D. : 1955, Monthly Notices Roy. Astron. Soc. 115, 236.

\section{DISCUSSION}

Schatzman: I am well aware that this description that I gave does not fit to all stars which are called novae. I would rather say that as far as novae are concerned, in the present situation there are processes which we understand and those we do not understand. and I am afraid I have spoken only of what $I$ understand. If I could give other examples, there are difficulties for example like nova Del which has been 
a very extraordinary object and many others (you mentioned the case of this nova where the double star has appeared). What $I$ think is possible is that we call novae a number of objects which are not exactly of the same physical state and the differences show up in the spectrum and in the light curve. I have concentrated, myself, either on the mechanism by which the outbursts can be produced in some cases rather than on the way one can derive from the observation the process which has taken place. 